\title{
An improved shuffled frog leaping algorithm and its application in 0-1 Knapsack Problem
}

\author{
Wenxiang Liu Yueguang Li
}

Gansu Normal University for Nationalities, Hezuo, gansu, 747000, China

\begin{abstract}
Keywords: Artificial fish swarm algorithm; Membrane computing; Optimization; 0-1 Knapsack Problem; Simulation
\end{abstract}

\begin{abstract}
NP complete problem. Shuffled frog leaping algorithm is applied successfully in many combinational optimization problems. Therefore, the paper introduces an improved shuffled frog leaping algorithm for solving 0-1 knapsack problem. It greatly reduces the searching time of shuffled frog leaping algorithm. It also effectively ameliorates the disadvantage of easily falling in local best of shuffled frog leaping algorithm. When the number of article is big, it also can obtain better effects. The simulation results show that the algorithm is more efficient.
\end{abstract}

\section{Introduction}

Shuffled frog leaping algorithm is proposed by Muzaffar Eusuff and Kevin Lansey on 2000 year, it is a swarm intelligence computation optimization algorithm [1]. It is used to solve discrete combinatorial optimization problem. As a new bionic intelligent optimization algorithm, it combined with memetic algorithms and particle swarm optimization algorithm, it has the advantages of two intelligence optimization algorithms. The features of the algorithm are concept simple, parameters is few, calculation speed is fast and global search ability is strong and easy to implement [2]. The main application of shuffled frog leaping algorithm is to solve multi objective optimization problems, such as water resources allocation, pier maintenance, workshop arranging flow engineering problem [3]. Domestic and foreign scholars have conducted a lot of research on it. Such as: Zhang joins "cognitive component" into the internal search strategy, improve the success rate of the algorithm for solving ability and jump out of local optimal solution [4]; Zhao Pengjun joins the attraction repulsion mechanism into the internal search strategy, effectively avoid the algorithm premature convergence [5]; Hatem E in the internal search strategy through the introduction of the "search accelerated factor", to improve the global searching ability of the algorithm [6].

Knapsack problem is a typical combinatorial optimization problem in operations research. It is a NP- complete problem, it is proposed by Markel and Hellman in the 50's of the last century [7]. The main idea of the knapsack problem is that a person has a lot of goods, the goods weights are not identical, the person must put some goods in the backpack. The weight of the goods is known, all possible goods are known, but the goods in the backpack are confidential, in addition additional backpack weight is limited. 0-1 knapsack problem is the most basic knapsack problems, other knapsack problem often can be converted into solving 0-1 knapsack problem.

\section{0-1 knapsack problem}

0-1 knapsack problem: given $\mathrm{n}$ goods and a backpack. The weight of goods $i$ is $w_{i}$, its value is $v_{i}$, the capacity of knapsack is c. Asked how to choose the goods of the backpack, so that the total value of goods of the backpack is maximum? The 0-1 knapsack problem can be formalized description: given $\quad \mathrm{c}$ or $\quad w_{i}>0 \quad v_{i}>0 \quad 1 \leq i \leq n \quad$. Asked to find an n-dimensional vector $\left(x_{1}, x_{2}, \ldots, x_{n}\right), x_{i} \in[0,1], 1 \leq i \leq n$, which makes $w_{1} x_{1}+w_{2} x_{2}+\ldots+w_{n} x_{n} \leq c$ is 
less than or equal to c, and $v_{1} x_{1}+v_{2} x_{2}+\ldots+v_{n} x_{n}$ reaches the maximum, 0-1 knapsack problem is a special integer planning problem [8]:

$$
\max \sum_{i=1}^{n} v_{i} x_{i} \quad \text { s.t. }\left\{\begin{array}{l}
\sum_{i=1}^{n} w_{i} x_{i} \leq c \\
x_{i} \in[0,1], 1 \leq i \leq n
\end{array}\right.
$$

\section{The mathematical model of shuffled frog leaping algorithm}

Shuffled frog leaping algorithm simulates the frog population when they are searching for food, according to ethnic classification of memes of information transfer. Shuffled frog leaping algorithm mainly includes two parts: local search and global information exchange. The following is a brief introduction to the mathematical model of shuffled frog leaping algorithm.

A randomly generated $\mathrm{F}$ frog consists of initial population, each frog expresses a feasible solution of problem, $U=\left(U^{1}, U^{2}, \ldots, U^{d}\right)$, calculate the frog individual fitness $f(i)$, where $\mathrm{d}$ denotes the dimension of the solution space. After the random generation of initial population, the individuals frog according to the fitness $f(i)$ in descending order is stored in the $X=\{U(i), f(i), i=1, \ldots, F\}$, then according to the specific principle to divide the whole frog population into m groups $Y^{1}, Y^{2}, \ldots, Y^{m}$, each group contains the $\mathrm{n}$ frogs, satisfy the following relations:

$$
\left.Y^{k}=\mid U(i)^{k}, f(i)^{k}, U(i)^{k}=U(k+m(i-1)), f(i)^{k}=f(k+m(i-1)), i=1, \ldots, n, n\right\}=1, \ldots, m ; F=m n
$$

In the frog population, the aim of various group perform local search strategy is to search the local optima in the different search direction, after a certain number of iterations, making the local optimal individuals in the population tends to the global optimal individual.

First of all, the frog population is divided into a plurality of groups, local search is carried out for each ethnic group, in order to avoid the frog individual into a local optimum prematurely, while speeding up the convergence process, in each group, according to specific principles choose a certain number frogs constitute the ethnic sub family group. For the frog population, with global best fitness of the solution is expressed as ${ }^{{ }_{g}}$; for each sub groups, with the best fitness of the solution is expressed as UB, the worst fitness solutions expressed as UW. The local search is carried out for each sub population, update strategy as following:

$$
\begin{aligned}
& S= \begin{cases}\min \left\{\left\{\operatorname{rand}\left(U_{B}-U_{W}\right)\right], S_{\max }\right\}, & U_{B}-U_{W} \geq 0 \\
\max \left\{\operatorname{rand}\left(U_{B}-U_{W}\right), S_{\max }\right\}, & U_{B}-U_{W}<0\end{cases} \\
& U_{q}=U_{W}+S
\end{aligned}
$$

Among them, $\mathrm{S}$ expresses the adjustment vector of frog individual, $S_{\text {mac }}$ represents the maximum step size of the frog allows to change.

Global information exchange helpful to collect local information of all kinds of group search, by meme transmission, obtain the search direction of new global optimal solution. After all populations conduct a certain number of local searches, various groups of frogs are mixed together, according to the fitness of $f(i)$ in descending order, the re division of population, which makes the meme information of the frog individual obtain the full transfer, then continue to conduct local search, so repeatedly until it is convergent, the algorithm stop.

\section{Improvement shuffled frog leaping algorithm for solving 0-1 Knapsack Problem}

Because the standard shuffled frog leaping algorithm, the individual frog position for continuous valued vector, algorithm cannot achieve scheduling updates, so the application of the shuffled frog leaping algorithm for the 0-1 Knapsack Problem needs to be constructed. 
According to the basic convergence properties of shuffled frog leaping algorithm, species formation thought is added in shuffled frog leaping algorithm, proposed an improved shuffled frog leaping algorithm. The specific steps of the algorithm are as follows:

(1) The initialization parameter (the frog population number is $m$, the frog number is $n$ in the population (total number of the frog $F=(\mathrm{mn})$, the frog number is $\mathrm{q}$ in sub groups, and the frog update iterations) ;

(2) Randomly generate F initial feasible solution, and calculate the fitness of the individual frogs;

(3) The frog individual is divided into $\mathrm{m}$ groups according to fitness in descending order, construct the sub groups;

(4) Local search. Update the frog individuals for each population group in the sub population groups in accordance with the method of this paper;

(5) Each frog individual mutation, such as the generation of new individuals better than original individual will replace the frog original individual into frog populations, re calculating the degree of adaptation;

(6) Determine whether the algorithm meets convergence conditions, if satisfied, output the optimal scheduling values and the corresponding scheduling solution, return to step (3).

\section{The analysis of simulation}

The data from the reference [7], using these data, the solution result can be seen in Table 1 . Compare with the algorithm of this paper and some algorithms in the literature [8], it is used to verify the effectiveness of the algorithm. In this paper, Run environment of algorithm is MATLAB (R2010b) under Win7 operating system. Parameters: the total number of the frog population $F=10$ n, the number of population $m=10$, the number of frog in the sub group $\mathrm{q}=2 \mathrm{n} / 3$, the iteration number $\mathrm{IT}=\mathrm{q}$, the biggest adjustment factor number $\mathrm{l} \max =\mathrm{n} / 2$.In the experiment, the generation method of example: random generation $w_{i}$ and $v_{i}$ in $1 \sim 100 .{ }^{c}=0.8\left(w_{1}+w_{2}+\ldots,+w_{n}\right)$.

$v=71,58,31,2,82,5,87,38,88,95,53,6,47,63,27,83,59,92,70,25,11,68,58,11,29,30,31,98,28,17,42,72,64,19,9$, $91,79,29,64,43,57,92,3,92,68,52,36,27,25,7,37,16,26,55,94,51,11,46,60,2,8,34,1,66,83,20,46,15,93,9,41,50,3$ $3,59,93,45,88,28,26,4,80,24,26,5,21,59,93,55,64,97,93,35,48,100,3,55,54,85,68,100, \mid 50,70,43,82,43,23,49,8$ $9,31,16,23,37,48,69,62,17,21,7,80,47,12,5,54,22,75,40,75,64,2,41,99,70,74,36,95,65,11,8,96,50,22,40,51,52$, $56,66,70,76,50,23,79,52,81,98,68,88,13,80,41,17,51,11,65,50,90,33,22,24,62,39,59,52,87,26,35,75,28,41,75$, $8,34,32,16,96,94,99,60,58,87,44,25,40,28,21,90,66,24,85,50,84, \| 20,42,62,21,51,100,1,29,90,18,2,38,98,59,1$ $5,3,79,34,56,57,21,58,53,58,55,97,100,41,99,18,2,70,11,40,32,81,38,85,73,41,65,23,75,91,67,29,2,99,59,27$, $80,10,42,46,50,95,60,62,19,2,43,56,5,6,61,60,91,58,74,6,76,35,80,80,21,91,66,67,1,8,59,5,57,42,74,33,41,77$ ,66,42,73,74,78,25,82,19,93,26,67,76,\|||83,90,36,36,58,14,64,65,19,98,39,55,4,90,12,78,42,44,51,28,98,62,8 $6,80,31,50,60,32,33,63,83,61,91,61,77,69,26,51,29,36,52,47,38,66,20,10,71,43,71,5,5,74,83,47,83,47,25,55$, $63,72,6,97,65,37,22,43,25,59,8,16,40,29,90,39,26,65,32,88,57,62,86,40,27,40,44,22,90,99,88,66,16,62,37,51$ ,38,30,2,62,3,32,30,33,24,71,58,100,25,77,63,50,97,59,11,74,44,50,6,12,32,61,42,7,44,28,51,52,26,65,55,43, $39,11,15,27,5,85,81,53,63,28,41,21,16,61,73,33,79,30,96,42,33,58,14,4,72,95,73,24,56,65,7,11,55,23,90,24$, $7,97,83,24,63,43,88,25,37,92,96,41,58,5,37,37,18,16,28,81,26,88,28,92,51,58,82,90,78,92,78,48,57,32)$.

$w=(54,29,78,77,71,42,80,97,6,37,77,60,30,65,28,83,99,23,99,54,100,2,11,80,5,39,95,41,17,65,42,33,21,5$ $9,46,27,38,92,63,10,70,84,55,44,51,47,41,6,98,40,49,48,63,16,66,40,79,76,84,22,11,13,54,55,9,68,36,71,54$, $76,47,21,10,17,10,28,76,68,9,33,30,49,35,49,87,76,34,9,42,12,63,15,22,14,35,93,41,83,73,34, \mid 42,18,55,55,5$ $1,62,69,38,30,53,59,88,20,75,79,81,96,7,38,12,18,72,57,47,76,91,9,72,44,28,81,42,28,44,13,35,19,44,55,98$, $38,29,14,97,91,45,7,71,16,33,6,76,33,81,91,42,96,70,2,17,41,28,85,19,38,78,45,88,38,87,94,34,26,18,1,85,7$ $1,82,44,42,72,80,60,25,12,64,91,25,8,76,38,53,59,8,12,91,95,45,77,39, \| 33,16,10,70,19,48,43,76,83,11,16,19$ ,88,73,26,83,80,46,81,21,37,35,43,6,21,94,84,81,26,70,34,12,30,56,30,31,41,41,66,96,52,59,46,29,90,37,30, $77,61,51,86,55,8,70,41,99,54,9,29,16,55,79,41,32,52,33,78,44,17,25,96,69,4,49,61,95,2,97,96,30,81,6,32,52$, $46,8,58,59,81,80,70,20,7,96,22,24,26,72,78,31, \||| 72,23,56,21,63,7,84,43,50,77,49,68,65,35,43,36,75,72,18,9$, $29,31,32,18,8,46,4,14,77,97,56,20,32,60,72,77,55,57,42,98,59,4,14,16,38,62,44,34,22,89,46,76,21,87,42,35$, $81,54,9,26,28,33,37,33,22,62,69,61,5,25,59,84,53,1,17,99,25,13,61,70,10,83,68,84,84,74,22,97,53,46,65,86$, $6,6,46,97,66,54,72,99,37,36,60,27,58,94,82,41,50,14,18,69,66,79,9,58,42,15,100,28,48,46,95,18,35,40,7,37$, $89,60,6,15,77,14,70,52,99,7,33,69,39,88,41,55,13,1,93,23,19,37,98,53,90,80,25,47,40,61,47,77,16,6,96,35,4$ 
Table 1 Comparison of the solution results of the algorithm in this paper and results in the literature [8]

\begin{tabular}{|c|c|c|c|c|c|c|c|}
\hline \multirow{2}{*}{$\begin{array}{c}\text { The } \\
\text { Maximum } \\
\text { capacity } \\
\text { of } \\
\text { backpack }\end{array}$} & \multirow[b]{2}{*}{$n$} & \multicolumn{3}{|c|}{$\begin{array}{l}\text { the algorithm } \\
\text { of this paper }\end{array}$} & \multicolumn{3}{|c|}{$\begin{array}{l}\text { Hybrid genetic } \\
\text { algorithm }\end{array}$} \\
\hline & & Algebraic & Value & Weight & Algebraic & Value & Weight \\
\hline 3852 & 100 & 97 & 4778 & 3852 & 2 & 4721 & 3850 \\
\hline 7876 & 200 & 163 & 9652 & 7868 & 7 & 9595 & 7867 \\
\hline 11780 & 300 & 149 & 14506 & 11770 & 12 & 14455 & 11779 \\
\hline 19803 & 500 & 95 & 23964 & 19803 & 16 & 23911 & 19803 \\
\hline
\end{tabular}

It can be seen from table 1, the solution results of improved shuffled frog leaping algorithm and the solution results of the literature [8] is the same, it show the effectiveness of improved shuffled frog leaping algorithm. Table 2 Compares the solution time of each generation of the algorithm in this paper and genetic algorithm in the literature [8], the result of solving time of this algorithm is far less than the solving time of genetic algorithm in the literature [8], especially the $n$ is large. It shows that the fast and effective of the algorithm of this paper.

Table 2 Comparison of the solution time of the algorithm in this paper and results in the literature [8]

\begin{tabular}{|c|c|c|}
\hline$n$ & the algorithm of this paper (s / g) & Hybrid genetic algorithm(s / g) \\
\hline 100 & 0.340 & 1.031 \\
\hline 200 & 2.767 & 9.323 \\
\hline 300 & 8.820 & 31.474 \\
\hline 500 & 38.265 & 152.503 \\
\hline 800 & 168.024 & 511.425 \\
\hline 1000 & 355.978 & 883.366 \\
\hline
\end{tabular}

\section{Conclusions}

The algorithm analysis and experimental results show that: the improved shuffled frog leaping algorithm for solving 0-1 knapsack problem greatly reduces the search time and effectively improves the defects of the algorithm easy to premature convergence to a non optimal solution, when the number of goods $(n)$ is large, it also can obtain better quality. Compared with some other methods for solving 0-1 knapsack problem, the algorithm achieves good results.

\section{References}

[1] EUSUFF M M, LANSEY K E. Water distribution network design using the shuffled frog leaping algorithm[A].World Water Congress[C].2001.

[2] LI Y H, ZHOU J Z, YANG J J, et al. Modified shuffled frog leaping algorithm based on threshold selection strategy[J]. Computer Engineering and Applications, 2007, 43(35):19-21.

[3] EUSUFF M M, LANSEY K E. Optimization of water distribution network design using the shuffled frog leaping algorithm[J]. J Water Resource Plan Manage, 2003, 129(3):10-25.

[4] Zhang Xuncai, Hu Xuemei. An improved shuffled frog leaping algorithm with cognitive behavior [C]. 2008.

[5] Zhao Pengjun, Liu Sanyang. The shuffled frog leaping algorithm in solving complex function optimization problems[J]. Application and Research of Computer. 2009, 26(7): 2435 - 2437.

[6]HATEM E, EMAD E, TAREK H, et al. Comparison of two evolutionary algorithms for optimization of bridge deck repairs [J]. Computer-Aided Civil and Infrastructure Engineering, 2006, 21:561-572.

[7]Ralph Merkle and Martin Hellman. Hiding Information and Signatures in Trapdoor Knapsacks[J]. IEEE Transactions on Information Theory,1978, 24(5): 525-530. 
[8] Song Haizhou, Wei Xuzhen. A hybrid genetic algorithm of solving 0-1 knapsack problem [J]. Journal of Huaqiao University (Natural Science), 2006, 27 (1): 16-19. 